\title{
Serious bacterial infections in febrile young children: Lack of value of biomarkers
}

\author{
M Karsas, MB ChB; P J Becker, PhD; R J Green, PhD, DSc \\ Department of Paediatrics and Child Health, School of Medicine, Faculty of Health Sciences, University of Pretoria, South Africa
}

Corresponding author: M Karsas (mkarsas@hotmail.com)

\begin{abstract}
Background. Serious infections in children are difficult to determine from symptoms and signs alone. Fever is both a marker of insignificant viral infection, as well as more serious bacterial sepsis. Therefore, seeking markers of invasive disease, as well as culture positivity for organisms, has been a goal of paediatricians for many years. In addition, the avoidance of unnecessary antibiotics is important in this time of emerging multiresistant micro-organisms.

Objective. To ascertain whether acute-phase reactant tests predict positive culture results.

Methods. A prospective, cross-sectional study over a 1-year period included all documented febrile children under the age of 5 years (with an axillary temperature $\geq 38^{\circ} \mathrm{C}$ ) who presented to Steve Biko Academic Hospital, Pretoria, with signs and symptoms of pneumonia, meningitis and/or generalised sepsis. Every child had clinical signs, chest radiograph findings, urine culture, blood testing (full blood count, C-reactive protein, procalcitonin) and blood culture results recorded.

Results. A total of 63 patients were enrolled, all of whom had an axillary temperature $\geq 38^{\circ} \mathrm{C}$. C-reactive protein, procalcitonin and white cell count did not predict the presence of positive blood culture or cerebrospinal fluid culture results, nor infiltrates on chest radiographs. No statistically significant correlations were found between the duration of hospital stay and the degree of fever $(p=0.123)$, white cell count $(p=0.611)$, C-reactive protein $(p=0.863)$ or procalcitonin $(p=0.392)$.

Conclusion. Biomarkers do not seem to predict severity of infection, source of infection, or duration of hospitalisation in children presenting to hospital with fever. The sample size is however too small to definitively confirm this viewpoint. This study suggests that clinical suspicion of serious infection and appropriate action are as valuable as extensive testing.
\end{abstract}

S Afr J Child Health 2016;10(1):33-36. DOI:10.7196/SAJCH.2016.v10i1.980

Young children often attend primary care institutions and emergency departments with acute infections. Most of these children have selflimiting conditions; however, a small proportion have serious or even life-threatening infections. This may be a source of anxiety for parents and may present a challenge to attending clinicians. ${ }^{[1]}$

There is reasonably good evidence for the diagnostic value of clinical features for certain conditions, namely pneumonia, and to a lesser degree, meningitis. However, little is known about the clinical features predictive of serious outcome for febrile children presenting with nonspecific symptoms and signs with no clear focus of infection. ${ }^{[1]}$

Various clinical tools have been developed in order to score febrile children as a means to predict the severity of illness, but these scores have been found to be nonspecific and of limited use in clinical practice, especially when used as positive predictors of serious bacterial infection (SBI). ${ }^{[2]}$

Fever in young children may represent both insignificant viral infections and SBI which is often not associated with a distinguishable source of infection. ${ }^{[3]}$ Seeking markers of invasive disease, as well as culture positivity for organisms, has been a goal of paediatricians for many years.

The evidence thus far, for multiple site testing, as well as multiple testing methods to detect SBIs in febrile young children, is unclear. In addition to selecting antibiotics for appropriate infections, the avoidance of unnecessary antibiotic use should also be considered important in this time of emerging multiresistant micro-organisms. This is an important aspect of antibiotic stewardship. ${ }^{[4]}$

\section{Methods}

A prospective, cross-sectional study over a 1-year period (1 December 2013 - 30 November 2014) of children presenting to Steve Biko Academic Hospital (SBAH), Pretoria, was conducted.
The study sampling strategy included paediatric patients requiring admission who met inclusion criteria and presented to casualty, the outpatients department or directly to the wards.

Approval was obtained from the Department of Paediatrics and Superintendent of SBAH, as well as the MMed and Ethics committees of the University of Pretoria. Informed consent was obtained from the parents/guardians of each participant.

SBAH is a large academic tertiary hospital located in Gauteng. It has two paediatric wards, with a maximum capacity of 56 beds, and one 7-bed paediatric ICU. All patients require referral from a clinic, medical practitioner or hospital. Patients not requiring tertiary care are down-referred to Tshwane District Hospital. The number of under-5 patients admitted per year is approximately $1400-1500$. Three-quarters $(\sim 1000)$ are admitted for subspecialist care and tend to be afebrile on admission; an eighth $(\sim 200)$ are neonates (excluded from the study), while $50 \%$ of the remaining $200-300$ patients have low-grade fevers $<38^{\circ} \mathrm{C}$ on admission (having been given prior antipyretics). Therefore the total number of children under 5 years of age expected for this cohort was 100 - 150 participants.

This study included all documented febrile patients (axillary temperature $\geq 38^{\circ} \mathrm{C}$ ) who presented to $\mathrm{SBAH}$ between the ages of 1 month and 5 years, with signs and symptoms of pneumonia, meningitis and/or generalised sepsis. Exclusion criteria for this study were children over the age of 5 years, neonates (less than 1 month of age), admission temperature $<38^{\circ} \mathrm{C}$, and children with exclusion criteria for a lumbar puncture (LP), namely focal neurological signs, papilloedema, readily deteriorating consciousness or Glasgow Coma Scale $(\mathrm{GCS})<8$, signs of raised intracranial pressure (falling pulse, rising blood pressure, dilating or poorly reacting pupils), continuous seizure activity, bleeding diathesis and neural-tube defects. ${ }^{[3]}$

Data collected included all the clinical, laboratory, radiological and microbiology findings. Data were collected on the day of 
admission, and updated as laboratory and microbiology results became available. In order to attempt to overcome the difference in clinical experience between admitting clinicians, specific signs and symptoms were listed in the data collection table that clinicians were instructed to document if present or absent on the day of admission. Patient age, gender, admission axillary temperature $\left({ }^{\circ} \mathrm{C}\right)$ and urine dipstick findings were also included in the captured data.

A 3-day cut-off value for the duration of hospitalisation was chosen for the purpose of this study as the median duration of hospitalisation for children at SBAH is 3 days. We expect those patients with severe illness to have a longer-than-median hospitalisation time.

A urine dipstick test was deemed positive in this study in the presence of leucocytes and/or nitrites. White cell count (WCC) was deemed to be increased if the WCC value was $>17 \times 10^{9} / \mathrm{L}$ in children aged $1-12$ months, and if $>15 \times 10^{9} / \mathrm{L}$ in children 13 months 5 years. ${ }^{[5]}$ Leucopenia was defined as WCC $<4 \times 10^{9} / \mathrm{L}$. A C-reactive protein (CRP) value was deemed positive/predictive of bacterial infection if CRP $>40 \mathrm{mg} / \mathrm{L}^{[6]}$ and procalcitonin $(\mathrm{PCT}$ ) was positive if $>0.2 \mu \mathrm{g} / \mathrm{L} .{ }^{[7]}$ Cerebrospinal fluid (CSF) findings were considered positive based either on biochemistry suggestive of meningitis, or on a positive CSF culture or PCR. ${ }^{[8]}$

Chest radiographs (CXRs) were interpreted by the lead investigator using the World Health Organization (WHO) CXR interpretation methodology. ${ }^{[9]}$ A CXR was deemed positive for hyperinflation in the presence of $>8$ visible posterior ribs ${ }^{[9]}$ This study also noted some combinations of both pneumonic changes and hyperinflation.

\section{Statistical analysis of data}

The primary data analysis focused on the proportion of children under the age of 5 years who were admitted to hospital and remained hospitalised for a period of 3 days or longer. Secondary data analysis was the agreement between the clinical picture and individual biomarkers, as well as among the individual biomarkers themselves.

All parametric data were analysed using a $t$-test and nonparametric data were analysed by means of Wilcoxon rank sum test using Stata-13 (StataCorp, USA).

The study was adequately powered.

\section{Results}

Sixty-three patients were enrolled (age range 1 month - 4.5 years), and $33(52.4 \%)$ were male.

All children whose HIV status was unknown or showed signs of being clinically immunocompromised were tested for HIV infection. Eight $(12.7 \%)$ patients were confirmed to be HIV-positive, in $5(62.5 \%)$ a significant organism was identified and in $2(25.0 \%)$ multiple significant organisms were cultured. Two (3.2\%) of the 63 patients were assessed as having malnutrition. Both patients were moderately acutely malnourished, and no organisms were cultured in either patient.

Thirty-seven (58.7\%) patients were hospitalised for $\geq 3$ days. The median temperature for all patients on admission (irrespective of duration of hospitalisation) was $38.4^{\circ} \mathrm{C}$ (range $38-40^{\circ} \mathrm{C}$ ). There was no statistical significance between temperature on admission and duration of stay $(p=0.123)$.

An organism was cultured from the blood in 13 (25.5\%) of the 51 patients in whom a blood culture was performed. All cultures were performed on admission. Commensal flora were isolated from $11(84.6 \%)$ of the 13 positive blood cultures (10 coagulase-negative Staphylococcus spp., 1 Micrococcus sp.). The two significant blood cultures isolated extended-spectrum beta-lactamase-producing Klebsiella pneumoniae and Salmonella Group D.

A significant organism was identified from any site in 25 (39.7\%) of the 63 patients included in this analysis. Sampled sites included blood $(8 / 51,15.7 \%)$, stool $(5 / 16,31.3 \%)$, urine $(7 / 34,20.6 \%)$, CSF
$(2 / 22,9.1 \%)$, respiratory secretions $(8 / 30,26.7 \%)$ or tissue $(3 / 3$, $100 \%)$. Among these 25 patients, significant multiple organisms were identified in $9(36.0 \%)$. Thus, significant multiple organisms were cultured in $14.3 \%$ of the total patients.

Nine subjects in total had an elevated WCC, and of these two $(22.2 \%)$ had positive insignificant blood cultures. None of the children with a decreased WCC had a positive blood culture. There was no correlation between temperature, WCC, CRP or PCT on admission and blood culture results $(p=0.521 ; p=0.919 ; p=0.186$; $p=0.833$, respectively). There was no correlation between duration of hospitalisation and blood culture results $(p=0.560)$.

Of the 63 enrolled patients, $54(85.7 \%)$ had a CXR. Of these 54 CXRs, 42 (77.8\%) revealed positive findings. Forty-six (73.0\%) of the 63 enrolled patients had respiratory symptoms and 35 (76.1\%) of them had positive CXR findings. In the 35 children with respiratory symptoms and positive CXR findings, 16 (45.7\%) had pneumonic changes, 11 (31.4\%) had hyperinflation on CXR, and pneumonic change with hyperinflation was found in $8(22.9 \%)$.

Of the 54 children who had CXRs, 30 (55.6\%) had pneumonic changes. Nasopharyngeal aspirates (NPAs) were collected from $10(33.3 \%)$ of the 30 children with pneumonic changes on CXR, $3(30.0 \%)$ of which were positive for respiratory syncytial virus (RSV) and 1 (10.0\%) was positive for Pneumocystis jiroveci (on immunoflourescence). Ten (33.3\%) of the 30 children with pneumonic changes on CXR had induced sputum specimens collected, one of which (in an HIV-positive child) was positive for acidalcohol-fast bacilli (AFB) and one positive for Candida albicans (HIV-negative child). Twenty (37.0\%) of the 54 children who had a CXR had positive findings of hyperinflation. NPAs were conducted in $5(25.0 \%)$ of the 20 children who had hyperinflated CXRs, and 2 of these were positive, 1 for RSV and 1 for parainfluenza virus type 3.

Seven $(70.0 \%)$ of the 10 children who had an elevated WCC and CXR performed had positive CXR findings. Both patients with a decreased WCC had radiographic changes. The proportion of all CXRs with positive findings $(n=42)$ with and without elevated or decreased WCC were compared with those with normal CXRs $(n=12)$. There was no correlation between WCC on admission and CXR findings of pneumonia or hyperinflation ( $p=0.145$ and $p=0.669$, respectively).

A positive WCC (elevated or decreased WCC) in combination with positive CSF findings was found in 1 of the 3 patients who had a positive WCC and an LP. These positive CSF findings were based on biochemistry suggestive of meningitis, even though they had a negative CSF culture and PCR. Twenty-two LPs were conducted in this cohort.

There was no correlation between WCC on admission and duration of hospital stay $(p=0.471)$.

CRP was measured in 59 (93.7\%) of the 63 patients enrolled in the study. A positive CRP (defined as a CRP $>40 \mathrm{mg} / \mathrm{L}$ ) ${ }^{[6]}$ was found in 25 (42.4\%) patients. Median (range) CRP was $31.0(<1$ to 336$) \mathrm{mg} / \mathrm{L}$. There was no correlation between CRP on admission and duration of stay $(p=0.863)$. A positive CRP in combination with positive CXR findings was found in $15(71.4 \%)$ of the 21 patients who had a positive CRP and had had a CXR. There was no correlation between CRP on admission and CXR findings of hyperinflation $(p=0.087)$ or pneumonia $(p=0.368)$.

A positive CRP in combination with a significant positive blood culture result was found in $2(9.5 \%)$ of the 21 patients who had a positive CRP and in whom a blood culture was performed. A positive CRP in combination with positive CSF findings was found in $6(60.0 \%)$ of 10 patients who had a positive CRP and in whom an LP was performed. Five out of the 6 positive CSF findings were based on biochemistry suggestive of meningitis. One was positive based on CSF PCR for enterovirus. 
PCT was measured in 25 (39.7\%) of the 63 enrolled patients. The clinical profile of these patients varied greatly, and $11(44.0 \%)$ were critically ill, requiring paediatric intensive care unit (PICU) admission. PCT was found to be positive $(>0.2 \mu \mathrm{g} / \mathrm{L})^{[7]}$ in $21(84.0 \%)$ of the 25 patients, $10(47.6 \%)$ of whom were admitted to ICU. Five $(50.0 \%)$ of these 10 died. Median (range) PCT was $5.7(0-728) \mu \mathrm{g} / \mathrm{L}$. Sixteen $(76.2 \%)$ of the 21 children with a positive PCT remained hospitalised for 5 days or longer; however, statistically there was no correlation between PCT on admission and duration of stay $(p=0.392)$.

A positive PCT in combination with positive CXR findings was found in $16(80.0 \%)$ of the 20 patients who had a positive PCT and in whom a CXR was performed. A positive PCT in combination with a significant positive blood culture result occurred in $1(4.8 \%)$ of the 21 patients who had a positive PCT and in whom a blood culture was performed. A positive PCT in combination with positive CSF findings occurred in $4(50.0 \%)$ of the 8 patients who had a positive PCT and in whom an LP was performed. Three (75.0\%) of the 4 were positive based on biochemistry suggestive of meningitis; 1 (25.0\%) was positive based on CSF PCR for enterovirus.

All of the children enrolled in the cohort had urine dipsticks evaluated at admission, and $12(19 \%)$ had positive urine dipsticks for leucocytes or nitrites. All of the urine specimens from children with positive urine dipsticks were culture-negative. Two (16.7\%) of the children with positive urine dipsticks had viral pathogens isolated on stool specimens (positive on stool ELISA): adenovirus $(n=1)$ and adenovirus and rotavirus $(n=1)$. In $8(15.7 \%)$ of the children with negative urine dipsticks an organism in the urine was cultured.

Of the 63 total enrolled patients, 11 (17.5\%) required PICU admission and 5 (7.9\%) patients died. Four of the five deaths occurred after the first 3 days of hospitalisation, with the median time to death being 10 days (range 2 - 20 days).

\section{Discussion}

The value of biomarkers for determining SBI in febrile children has revealed conflicting results. Some studies suggest that individual tests perform better than others or than clinical judgement of bacterial $v$. viral infection, while other studies do not. ${ }^{[3,10-13]}$

The insensitivity of routine microbiological methodologies in identifying bacterial infections, particularly bacteraemia, is well described, and molecular diagnostic techniques may in fact be superior to acute-phase reactants in detecting SBI in children. ${ }^{[14]}$ In a study performed to understand the epidemiology of childhood bacterial diseases, including invasive pneumococcal disease (IPD), screening criteria were used to identify children aged less than 5 years of age who had signs and symptoms of SBI. ${ }^{[14]}$ The study concluded that PCR and antigen testing increased the sensitivity of detection and provided a more precise estimation of the burden of invasive bacterial disease than bacterial culture. ${ }^{[14]}$ This study does not however argue against the use of CRP, PCT and other inflammatory biomarkers.

CXRs and their correlating biomarkers have been shown to have good test sensitivity for pulmonary disease. ${ }^{[9]}$ In our study, all patients with a decreased WCC, $80 \%$ of those with a positive PCT, $71.4 \%$ with a positive CRP and $70 \%$ with an elevated WCC had positive CXR changes. However, these biomarkers cannot be used to distinguish pneumonic changes from hyperinflation.

The results from the analysis of data collected from febrile young children presenting to SBAH reveal that fever or degree of fever does not predict severity of infection, nor source of infection, nor duration of hospitalisation. Degree of fever does not predict biomarkers for bacterial infection (WCC, CRP and PCT). Elevated biomarkers are not related to duration of hospitalisation nor do they predict a positive blood culture. However, it is important to keep in mind that the sensitivity of blood cultures is known to be low, and this is further impacted by the inordinately high $(11 / 51,21.6 \%)$ culture contamination rate at the study facility. The patient's clinical picture may be more valuable than CRP/WCC when deciding on choice of antibiotics and whether or not an organism is cultured. CRP/WCC cannot be used to predict SBIs in febrile young children and therefore cannot be used to decide on choice of antibiotics.

A low WCC is just as significant a marker for sepsis in children as a high WCC. Literature suggests that the risk of bacteraemia increases from $0.5 \%$ if the WCC is $<15 \times 10^{9} / \mathrm{L}$ to more than $18 \%$ if WCC is $>30 \times 10^{9} / \mathrm{L}^{[15]}$ Findings from the literature ${ }^{[15]}$ and our study reveal that more children with a WCC abnormality secondary to sepsis present with a high WCC. Twelve (85.7\%) of the 14 patients with WCC abnormalities had an increased WCC.

There was a wide range of CRP findings and CRP was specifically unhelpful in predicting infection severity when using cut-off values suggested by the literature. ${ }^{[6]}$

PCT is an expensive biomarker and therefore not usually performed on febrile children presenting to casualty; it is usually reserved for patients hospitalised in the PICU. Fourteen (56\%) of the 25 patients in whom a PCT was performed were stable with various illnesses while 11 (44\%) were critically ill requiring ICU admission. The limited number of PCTs done, because of the cost restraint, as well as the wide variability of the positive PCT values $(>0.2$; range $3-728 \mu \mathrm{g} / \mathrm{L}$ ) obtained, makes it difficult to interpret data. As $50 \%$ of patients with positive CSF results also had a high PCT, it may possibly have a predictive role with regard to meningitis; however, the cohort is too small to confirm this finding.

The sterility of the blood culture techniques used in this study is questionable. Eleven (21.6\%) of the 51 blood cultures that were taken cultured a commensal. Therefore, it can be concluded that one-fifth of blood culture specimens are not taken using the correct sterile procedures. This makes it difficult to interpret findings regarding true disease-causing pathogens $v$. commensal organisms. This problem with specimen collection may reflect, too, the significant number of negative tests.

The results of this study question the validity of urine dipsticks or the method of reading the test. All urine dipsticks are performed in the ward and read by nursing or medical staff; this may call into question the reliability of the staff's performance and interpretation of the test. Formal urine sample tests are superior to urine dipsticks. ${ }^{[16]}$ This study revealed that a negative urine dipstick does not rule out a urinary tract infection (UTI) and a positive urine dipstick does not confirm a UTI.

Blood tests such as WCC, CRP and PCT may be of more value in assessing response to treatment, rather than predicting the severity of sepsis. Relying on them to withhold or start antibiotic therapy is not prudent. The choice of whether to use antibiotic therapy or when to start treatment should rather be based on clinical judgement, as is invariably the case in the clinical environment in busy hospitals in South Africa, where empiric antibiotic use is most often implemented before laboratory test results have become available.

Routine CXRs are not justified unless a bacterial pneumonia is clinically suspected. This study is a poor example of when to use CXRs judiciously. CXRs were conducted in cases of generalised sepsis, upper respiratory tract infections, viral pneumonias and acute gastroenteritis, without supportive clinical signs of bacterial pneumonia.

If there is any concern for bacterial meningitis, then an LP is still mandatory. CSF Gram stain, bacterial antigen and culture are extremely useful markers to aid in diagnosis, whereas there is no consensus regarding CSF cell count and biochemistry in differentiating between viral and bacterial meningitis. The empiric use of antibiotics in this case should also be based on clinical judgment and not biomarker testing, until the results of the Gram stain, bacterial antigen and culture are available. 
This study was not without limitations. The small, possibly poorly representative sample size, with no control group and erratic sampling methods (owing to clinical discretion and cost constraints), reflects the large number of febrile children presenting to hospital with undocumented or low-grade fever.

\section{Conclusion}

Fever or degree of fever does not predict severity of infection, nor source of infection, nor duration of hospitalisation. Elevated biomarkers (WCC, CRP, and PCT) are not related to duration of hospital stay; they do not predict positive culture results nor identification of significant organisms. Thus, WCC, CRP, and PCT were not shown to be effective in predicting SBIs in febrile children under 5 years of age. This study suggests that clinical suspicion of serious infection and appropriate action are as valuable as extensive testing.

\section{References}

1. Thompson MJ, van den Bruel A. Diagnosing serious bacterial infection in febrile children. BMJ 2010;340:2062. [http://dx.doi.org/10.1136/bmj.c2062]

2. Bang A, Chaturvedi P. Yale Observational scale for prediction of bacteremia in febrile children. Indian J Pediatr 2009;76(6):599-604. [http://dx.doi. org/10.1007/s12098-009-0065-6]

3. Powell KR. Fever without a focus. In: Kliegman RM, Behrman RE, Jenson HB, Stanton BF, eds. Nelson Textbook of Paediatrics. 18th ed. New York: Saunders, 2007:1087-1089. [http://dx.doi.org/10.1016/b978-1-4377-06437.00096-6]

4. Squire EN Jr, Reich HM, Merenstein GB, Favara BE, Todd JK. Criteria for the discontinuation of antibiotic therapy during presumptive treatment of suspected neonatal infection. Pediatr Infect Dis 1982;1(2):85-90. [http://dx.doi. org/10.1097/00006454-198203000-00004]

5. Crisp S, Rainbow J. Emergencies in Paediatrics and Neonatology. 2nd ed. London: Oxford University Press, 2013. [http://dx.doi.org/10.1093/ med/9780199605538.001.0001]
6. Korppi M, Kröger L. C-reactive protein in viral and bacterial respiratory infection in children. Scand J Infect Dis 1993;25(2):207-213. [http://dx.doi. org/10.3109/00365549309008486]

7. Page AL, de Rekeneire N, Sayadi S, et al. Diagnostic and prognostic value of procalcitonin and C-reactive protein in malnourished children. Pediatrics 2014;133(2):363-370. [http://dx.doi.org/10.1542/peds.2013-2112]

8. Boyels $\mathrm{TH}$, Bamford $\mathrm{C}$, Bateman $\mathrm{K}$, et al. Guidelines for the management of acute meningitis in children and adults in South Africa. S Afr J Epidemiol Infect 2013;28(1):5-15. http://www.sajei.co.za/index.php/SAJEI/article/view/528 (accessed 10 February 2016).

9. Cherian T, Mulholland EK, Carlin JB, et al. Standardized interpretation of paediatric chest radiographs for the diagnosis of pneumonia in epidemiological studies. Bulletin of the World Health Organization 2005;83(5):353-359. http:// www.who.int/bulletin/volumes/83/5/353.pdf (accessed 10 February 2016).

10. Manzano S, Bailey B, Gervaix A, Cousineau J, Delvin E, Girodias JB. Markers for bacterial infection in children with fever without source. Arch Dis Child 2011;96(5):440-446. [http://dx.doi.org/10.1136/adc.2010.203760]

11. Andreola B, Bressan S, Callegaro S, Liverani A, Plebani M, Da Dalt L. Procalcitonin and C-reactive protein as diagnostic markers of severe bacterial infections in febrile infants and children in the emergency department. Pediatr Infect Dis J 2007;26(8):672-677. [http://dx.doi.org/10.1097/inf.0b013e31806215e3]

12. Thayyil S, Shenoy M, Hamaluba M, Gupta A, Frater J, Verber IG. Is procalcitonin useful in early diagnosis of serious bacterial infections in children? Acta Paediatr 2005;94(2):155-158. [http://dx.doi.org/10.1111/j.1651-2227.2005. tb01883.x]

13. Rudinsky SL, Carstairs KL, Reardon JM, Simon LV, Riffenburgh RH, Tanen DA. Serious bacterial infections in febrile infants in the post-pneumococcal conjugate vaccine era. Acad Emerg Med 2009;16(7):585-590. [http://dx.doi. org/10.1111/j.1553-2712.2009.00444.x]

14. Anh DD, Kilgore PE, Slack MP, et al. Surveillance of pneumococcal-associated disease among hospitalized children in Khanh Hoa Province, Vietnam. Clin Infect Dis 2009;48 Suppl 2:S57-64. [http://dx.doi.org/10.1086/596483]

15. Luszczak M. Evaluation and management of infants and young children with fever. Am Fam Phys 2001;64(7):1219-1226. http://www.aafp.org/ afp/2001/1001/p1219.html (accessed 10 February 2016).

16. Welsh A, ed. Urinary Tract Infection in Children: Diagnosis, Treatment and Longterm Management. NICE Clinical Guideline. National Collaborating Centre for Women's and Children's Health 2007:52-63. http://www.nice.org.uk/guidance/ cg54/evidence/full-guideline-196566877 (accessed 10 February 2016). 